\title{
Warped product semi-slant submanifolds in locally conformal Kaehler manifolds II
}

\author{
Koji Matsumoto
}

\begin{abstract}
In 1994 N. Papaghiuc introduced the notion of semi-slant submanifold in a Hermitian manifold which is a generalization of $C R$ - and slant-submanifolds, [4], [10]. In particular, he considered this submanifold in Kaehlerian manifolds, [13]. Then, in 2007, V. A. Khan and M. A. Khan considered this submanifold in a nearly Kaehler manifold and obtained interesting results, [9].

Recently, we considered semi-slant submanifolds in a locally conformal Kaehler manifold and we gave a necessary and sufficient conditions of the two distributions (holomorphic and slant) be integrable. Moreover, we considered these submanifolds in a locally conformal Kaehler space form.

In the last paper, we defined 2-kind warped product semi-slant submanifolds in almost hermitian manifolds and studied the first kind submanifold in a locally conformal Kaehler manifold. Using Gauss equation, we derived some properties of this submanifold in an locally conformal Kaehler space form, [3], [11].

In this paper, we consider same submanifold with the parallel second fundamental form in a locally conformal Kaehler space form. Using Codazzi equation, we partially determine the tensor field $P$ which defined in (1.2), see Theorem 4.1. Finally, we show that, in the first type warped product semi-slant submanifold in a locally conformal space form, if it is normally flat, then the shape operators $A$ satisfy some special equations, see Theorem 5.2.
\end{abstract}

Анотація. В 1994 році Н Папагіук ввів поняття напівпохилого (semislant) підмноговиду що є зануреним у ермітовий многовид. Такі підмноговиди є узагальненням $C R$-підмноговидів та похилих (slant) підмноговидів. На цих многовидах дотичне розшарування є прямою сумою голоморфного та похилого розподілів, [4], [10]. Зокрема, він розглядав таку структуру як підмноговид келерового многовиду, [13]. Згодом, у 2007 році В. А. Хан, та М. А. Хан досліджували такий підмноговид, занурений у наближено келеровий многовид, та отримали цікаві результати, [9].

2010 Mathematics Subject Classification:

$U D C$

Keywords: Locally conformal Kaehler manifold, slant distribution, semi-slant submanifold, warped product semi-slant submanifold 
Нещодавно, автором було досліджено напівпохилі підмноговиди занурені у локально конформно-келерові многовиди і отримано необхідні та достатні умови інтегровності обох розподілів (голоморфного та похилого). Крім того, вивчались напівпохилі підмноговиди локально конформно-келерової просторової форми.

В останній статті автором введено два типи напівпохилих підмноговидів, що є викривленими добутками, занурених у майже ермітові многовиди та досліджено підмноговиди першого типу в локально конформнокелерових многовидах. Використовуючи рівняння Гауса, ми отримали деякі властивості такого підмноговиду локально конформно-келерової просторової форми, [3], [11].

В представлені роботі роглядаються напівпохилі підмноговиди локально конформно-келерових просторових форм, які мають паралельну другу фундаментальну форму. За допомогою рівняння Кодацці знайдено вигляд тензору $P$, що визначений у (1.2) (див. Теорему 4.1). Отримано умови на оператор Вейнгартена $A$ за яких напівпохилий підмноговид локально конформно-келерової просторової форми є викривленим добутком з плоскою нормальною зв'язністю, (див. Теорему 5.2).

\section{INTRODUCTION}

A Hermitian manifold $\tilde{M}$ with structure $(J, \tilde{g})$ is called a locally conformal Kaehler (an l.c.K. manifold) if each point $x \in \tilde{M}$ has an open neighborhood $U$ with a differentiable function $\rho: U \rightarrow \mathbb{R}$ such that $\tilde{g}^{*}=\left.e^{-2 \rho} \tilde{g}\right|_{U}$ is a Kaehlerian metric on $U$, that is, $\nabla^{*} J=0$, where $J$ is an almost complex structure, $\tilde{g}$ is a Hermitian metric, $\nabla^{*}$ is the covariant differentiation with respect to $\tilde{g}^{*}$, and $\mathbb{R}$ is a real number space, [14], [10].

Proposition 1.1. A Hermitian manifold $\tilde{M}$ with structure $(J, \tilde{g})$ is an l.c.K.-manifold if and only if there exists a global closed 1-form $\alpha$, called Lee form, satisfying

$$
\left(\tilde{\nabla}_{V} J\right) U=-\tilde{g}\left(\alpha^{\sharp}, U\right) J V+\tilde{g}(V, U) \beta^{\sharp}+\tilde{g}(J V, U) \alpha^{\sharp}-\tilde{g}\left(\beta^{\sharp}, U\right) V
$$

for any $V, U \in T \tilde{M}$, where $\tilde{\nabla}$ denotes the covariant differentiation with respect to $\tilde{g}, \alpha^{\sharp}$ is the dual vector field of $\alpha$, the 1-form $\beta$ is defined by $\beta(X)=-\alpha(J X), \beta^{\sharp}$ is the dual vector field of $\beta$, and $T \tilde{M}$ is the tangent bundle of $\tilde{M}$.

An l.c.K.-manifold $\tilde{M}(J, \tilde{g}, \alpha)$ is called an l.c.K.-space form if it has a constant holomorphic sectional curvature. Then, [9], the Riemannian curvature tensor $\tilde{R}$ with respect to $\tilde{g}$ of an l.c.K.-space form with the constant holomorphic sectional curvature $c$ is given by the following formula:

$$
4 \tilde{R}(X, Y, Z, W)=c\{\tilde{g}(X, W) \tilde{g}(Y, Z)-\tilde{g}(X, Z) \tilde{g}(Y, W)+
$$




$$
\begin{gathered}
+\tilde{g}(J X, W) \tilde{g}(J Y, Z)-\tilde{g}(J X, Z) \tilde{g}(J Y, W)- \\
\quad-2 \tilde{g}(J X, Y) \tilde{g}(J Z, W)\}+ \\
+3\{P(X, W) \tilde{g}(Y, Z)-P(X, Z) \tilde{g}(Y, W)+ \\
\quad+\tilde{g}(X, W) P(Y, Z)-\tilde{g}(X, Z) P(Y, W)\}+ \\
+P(J X, W) \tilde{g}(J Y, Z)+P(J X, Z) \tilde{g}(J Y, W)+ \\
-\tilde{g}(J X, W) P(J Y, Z)+\tilde{g}(J X, Z) P(J Y, W)+ \\
+2\{P(J X, Y) \tilde{g}(J Z, W)+\tilde{g}(J X, Y) P(J Z, W)\}
\end{gathered}
$$

for any $X, Y, Z, W \in T \tilde{M}$, where $P$ is defined by

$$
P(X, Y)=-\left(\tilde{\nabla}_{X} \alpha\right) Y-\alpha(X) \alpha(Y)+\frac{1}{2}\|\alpha\|^{2} \tilde{g}(X, Y),
$$

for any $X, Y \in T \tilde{M}$, where $\|\alpha\|$ is the length of the Lee form $\alpha$ with respect to $g$.

Let $(M, g)=M_{1} \otimes_{f} M_{2}$ be a warped product Riemannian manifold of $\left(M_{1}, g_{1}\right)$ and $\left(M_{2}, g_{2}\right)$ with a warping function $f,[12]$. Then $g$ is given by

$$
g(U, V)=e^{f^{2}} g_{1}\left(\pi_{1 *} U, \pi_{1 *} V\right)+g_{2}\left(\pi_{2 *} U, \pi_{2 *} V\right)
$$

for any $U, V \in T M$, where $\pi_{1}$ (resp. $\pi_{2}$ ) denotes the projection operator of $M$ to $M_{1}\left(\operatorname{resp} . M_{2}\right)$ and $\pi_{1 *}\left(\operatorname{resp} \pi_{2 *}\right)$ is the differential of $\pi_{1}$ (resp. $\left.\pi_{2}\right)$.

Let $\nabla, \nabla_{1}$ and $\nabla_{2}$ be the covariant differentiation with respect to $g, g_{1}$ and $g_{2}$, respectively. Then we have from (1.3)

$$
\begin{aligned}
\nabla_{X} Y & =\nabla_{1 X} Y-f^{2} e^{f^{2}} g_{1}(X, Y)\left(\triangle_{2} \log f\right), \\
\nabla_{X} Z & =\nabla_{Z} X=f^{2}(Z \log f) X \\
\nabla_{Z} W & =\nabla_{2 Z} W
\end{aligned}
$$

for any $X, Y \in T M_{1}$ and $Z, W \in T M_{2}$, where we put

$$
\left(\triangle_{2} \log f\right)(Z)=\left(d_{2} \log f\right)(Z) .
$$

By virtue of (1.3) and (1.4), the curvature tensor form $R(X, Y, Z, W)$ is given by

$$
\begin{aligned}
& R\left(X_{1}, X_{2}, X_{3}, X_{4}\right)=e^{f^{2}}\left[R_{1}\left(X_{1}, X_{2}, X_{3}, X_{4}\right)-\right. \\
& -f^{4} e^{f^{2}}\left\|\nabla_{2} \log f\right\|^{2}\left\{g_{1}\left(X_{1}, X_{4}\right) g_{1}\left(X_{2}, X_{3}\right)-\right. \\
& \left.\left.-g_{1}\left(X_{1}, X_{3}\right) g_{1}\left(X_{2}, X_{4}\right)\right\}\right] \\
& R\left(X_{1}, Z_{1}, Z_{2}, X_{2}\right)=-f^{2} e^{f^{2}}\left\{\left(2+f^{2}\right)\left(Z_{2} \log f\right)\left(Z_{1} \log f\right)+\right. \\
& \left.+\nabla_{2 Z_{1}} \nabla_{2 Z_{2}} \log f\right\} g_{1}\left(X_{1}, X_{2}\right) \\
& R\left(Z_{1}, Z_{2}, Z_{3}, Z_{4}\right)=R_{2}\left(Z_{1}, Z_{2}, Z_{3}, Z_{4}\right) \text {, }
\end{aligned}
$$




$$
\text { Other }=0
$$

for any $X_{1}, X_{2}, X_{3}, X_{4} \in T M_{1}$ and $Z_{1}, Z_{2}, Z_{3}, Z_{4} \in T M_{2}$, where $R_{1}$ and $R_{2}$ be the Riemannian curvature forms with respect to $g_{1}$ and $g_{2}$, respectively. Next, using (1.5), the Ricci tensor $\rho(U, V)$ is separated as

$$
\begin{aligned}
& \rho\left(X_{1}, X_{2}\right)=\rho_{1}\left(X_{1}, X_{2}\right)-f^{2} e^{f^{2}}\{(2+\left.n_{1} f^{2}\right)\left\|\nabla_{2} \log f\right\|^{2}+ \\
&\left.+\delta_{2} d_{2} \log f\right\} g_{1}\left(X_{1}, X_{2}\right), \\
& \rho\left(X_{1}, Z_{1}\right)=0, \\
& \rho\left(Z_{1}, Z_{2}\right)=\rho_{2}\left(Z_{1}, Z_{2}\right)-n_{1} f^{2}\left\{\left(2+f^{2}\right)\left(\nabla_{2 Z_{1}} \log f\right)\left(\nabla_{2 Z_{2}} \log f\right)+\right. \\
&\left.+\nabla_{2 Z_{1}} \nabla_{2 Z_{2}} \log f\right\},
\end{aligned}
$$

where $\rho_{1}$ (resp. $\rho_{2}$ ) denotes the Ricci tensor with respect to $g_{1}$ (resp. $g_{2}$ ). Finally, if we respectively put $\tau, \tau_{1}$ and $\tau_{3}$ the scalar curvature with respect to $g, g_{1}$ and $g_{2}$. It easily follows that

$$
\tau=e^{f^{2}} \tau_{1}+\tau_{2}-\left(n_{1}-1\right) n_{1} f^{4}\left\|\nabla_{2} \log f\right\|^{2}
$$

\section{Semi-Slant-Submanifolds in An Almost Hermitian manifold.}

In generall, for a Riemannian manifold $(\tilde{M}, \tilde{g})$ and its Riemannian submanifold $(M, g)$ we know the Gauss and Weingarten formulas

$$
\tilde{\nabla}_{X} Y=\nabla_{X} Y+\sigma(X, Y), \quad \tilde{\nabla}_{X} N=-A_{N} X+\nabla^{\perp}{ }_{X} N
$$

for all $X, Y \in T M$ and $N \in T^{\perp} M$, where $\nabla$ is the covariant differentiation with respect to $g, \sigma$ is the second fundamental form, and $A_{N}$ is the shape operator or the fundamental tensor of Weingarten with respect to $N$ and $\nabla^{\perp}$ is normal connection, [5], [6]. Also the following identity holds true:

$$
\tilde{g}\left(A_{N} Y, X\right)=\tilde{g}(\sigma(Y, X), N) .
$$

The Codazzi equation and the Ricci equation are respectively given by

$$
\begin{aligned}
\tilde{R}\left(U, V, W, N_{1}\right) & =\tilde{g}\left(\left(\bar{\nabla}_{U} \sigma\right)(V, W)-\left(\bar{\nabla}_{V} \sigma\right)(U, W), Z\right), \\
\tilde{R}\left(U, V, N_{1}, N_{2}\right) & =R^{\perp}\left(U, V, N_{1}, N_{2}\right)-\tilde{g}\left(\left[A_{N_{1}}, A_{N_{2}}\right] U, V\right)
\end{aligned}
$$

for all $U, V, W, Z \in T M$ and $N_{1}, N_{2} \in T^{\perp} M$, where $R^{\perp}$ is the normal curvature tensor, and

$$
\left(\bar{\nabla}_{U} \sigma\right)(V, W)=\nabla_{U}^{\perp} \sigma(V, W)-\sigma\left(\nabla_{U} V, W\right)-\sigma\left(V, \nabla_{U} W\right) .
$$

The second fundamental form $\sigma$ is called parallel if it satisfies $\bar{\nabla} \sigma=0$ identically. Also a submanifold $M$ is said to be totally geodesic whenever $\sigma=0$ on $M$. 
Let $(M, g)=\left(M_{1}, g_{1}\right) \otimes_{f}\left(M_{2}, g_{2}\right)$ be a warped product submanifold of $(\tilde{M}, \tilde{g})$. Then the induced metric tensor $g$ of $\tilde{g}$ is given by

$$
g(U, V)=e^{f^{2}} g_{1}\left(\pi_{1 *} U, \pi_{1 *} V\right)+g_{2}\left(\pi_{2 *} U, \pi_{2 *} V\right)
$$

for any $U, V \in T M$. A warped product submanifold $M$ is called $M_{1}$ (resp. $M_{2}$ ) geodesic if the second fundamental form satisfies $\sigma(X, Y)=0$ (resp. $\sigma(Z, W)=0$ ) for all $X, Y \in T M_{1}$ and $Z, W \in T M_{2}$. Moreover $M$ is said to be mixed totally geodesic if the second fundamental form $\sigma$ satisfies $\sigma(X, Z)=0$ for all $X \in T M_{1}$ and $Z \in T M_{2}$.

By virtue of (1.5) and the Gauss equation, we have

$$
\begin{aligned}
& \tilde{R}\left(X_{1}, X_{2}, X_{3}, X_{4}\right)=e^{f^{2}}\left\{R_{1}\left(X_{1}, X_{2}, X_{3}, X_{4}\right)-\right. \\
& -f^{4} e^{f^{2}}\|\log f\|^{2}\left(g_{1}\left(X_{1}, X_{4}\right) g_{1}\left(X_{2}, X_{3}\right)-g_{1}\left(X_{1}, X_{3}\right) g_{1}\left(X_{2}, X_{4}\right)\right\}+ \\
& +\tilde{g}\left(\sigma\left(X_{1}, X_{4}\right), \sigma\left(X_{2}, X_{3}\right)\right)-\tilde{g}\left(\sigma\left(X_{1}, X_{3}\right), \sigma\left(X_{2}, X_{4}\right)\right), \\
& \tilde{R}\left(X_{1}, X_{2}, X_{3}, Z_{1}\right)=\tilde{g}\left(\sigma\left(X_{1}, Z_{1}\right), \sigma\left(X_{2}, X_{3}\right)\right)-\tilde{g}\left(\sigma\left(X_{1}, X_{3}\right), \sigma\left(X_{2}, Z_{1}\right)\right) \text {, } \\
& \tilde{R}\left(X_{1}, X_{2}, Z_{1}, Z_{2}\right)=\tilde{g}\left(\sigma\left(X_{1}, Z_{2}\right), \sigma\left(X_{2}, Z_{1}\right)\right)-\tilde{g}\left(\sigma\left(X_{1}, Z_{1}\right), \sigma\left(X_{2}, Z_{2}\right)\right), \\
& \tilde{R}\left(X_{1}, Z_{1}, Z_{2}, X_{2}\right)=-f^{2} e^{f^{2}}\left\{\left(2+f^{2}\right)\left(Z_{1} \log f\right)\left(Z_{2} \log f\right)+\right. \\
& \left.+\nabla_{2 Z_{2}} \nabla_{2 Z_{1}} \log f\right\} g_{1}\left(X_{1}, X_{2}\right)+\tilde{g}\left(\sigma\left(X_{1}, X_{2}\right), \sigma\left(Z_{1}, Z_{2}\right)\right)- \\
& -\tilde{g}\left(\sigma\left(X_{1}, Z_{2}\right), \sigma\left(Z_{1}, X_{2}\right)\right) \text {, } \\
& \tilde{R}\left(X_{1}, Z_{1}, Z_{2}, Z_{3}\right)=\tilde{g}\left(\sigma\left(X_{1}, Z_{3}\right), \sigma\left(Z_{1}, Z_{2}\right)\right)-\tilde{g}\left(\sigma\left(X_{1}, Z_{2}\right), \sigma\left(Z_{1}, Z_{3}\right)\right) \text {, } \\
& \tilde{R}\left(Z_{1}, Z_{2}, Z_{3}, Z_{4}\right)=R_{2}\left(Z_{1}, Z_{2}, Z_{3}, Z_{4}\right)+\tilde{g}\left(\sigma\left(Z_{1}, Z_{4}\right), \sigma\left(Z_{2}, Z_{3}\right)\right)- \\
& -\tilde{g}\left(\sigma\left(Z_{1}, Z_{3}\right), \sigma\left(Z_{2}, Z_{4}\right)\right) \text {, }
\end{aligned}
$$

for all $X_{1}, X_{2}, X_{3}, X_{4} \in T M_{1}$ and $Z_{1}, Z_{2}, Z_{3}, Z_{4} \in T M_{2}$.

For a vector field $U \in T M$, the angle between $J U$ and $T M$ is called the Wirtinger angle of $U$.

A differentiable distribution $\mathcal{D}^{\theta}: x \rightarrow \mathcal{D}^{\theta}{ }_{x}$ on $M$ is said to be a slant one if for each $U_{x} \in \mathcal{D}^{\theta}{ }_{x}$, the Wirtinger angle of $U_{x}$ is constant $(=\theta)$ for any $x \in M$. In this case, the Wirtinger angle is said to be the slant angle. In particular, if $T M$ is slant, then the submanifold is called slant as well. A slant submanifold is holomorphic (resp. totally real) if its slant angle $\theta=0$ (resp. $\theta=\frac{\pi}{2}$ ). A slant submanifold is said to be proper if it is not holomorphic nor totally real.

A submanifold $M$ in $\tilde{M}$ is called semi-slant if there exists a differentiable distribution $\mathcal{D}: x \rightarrow \mathcal{D}_{x} \subset T_{x} M$ on $M$ satisfying the following conditions 
(i) $\mathcal{D}$ is holomorphic, i.e., $J \mathcal{D}_{x}=D_{x}$ for each $x \in M$ and

(ii) the complementary orthogonal distribution $\mathcal{D}^{\theta}: x \rightarrow \mathcal{D}^{\theta}{ }_{x} \subset T_{x} M$ is slant with slant angle $\theta$, where $T_{x} M$ means the tangent vector space of $M$ at $x$, [7].

Remark 2.1. A semi-slant submanifold is a $C R$-submanifold if the slant angle is equal to $\frac{\pi}{2}$, e.g. [1], [2], [8].

In a submanifold $M$ of an almost Hermitian manifold $\tilde{M}(J, \tilde{g})$, for all $U \in T M$ and $\xi \in T^{\perp} M$, we write

$$
J U=T U+F U, \quad J \xi=t \xi+f \xi,
$$

where $T U$ (resp. $F U$ ) is the tangential (resp. normal) component of $J U$ and $t \xi$ (resp. $f \xi$ ) is the tangential (resp. normal) component of $J \xi$.

Then one can easily check the following relations:

$$
\begin{aligned}
T^{2}+t F & =-I, & f^{2}+F t & =-I \\
F T+f F & =0, & t F+T t & =0 .
\end{aligned}
$$

For a semi-slant submanifold $M$ of an almost Hermitian manifold $\tilde{M}$ the tangent bundle $T M$ and the normal bundle $T^{\perp} M$ of $M$ are decomposed as

$$
T M=\mathcal{D} \oplus \mathcal{D}^{\theta}, \quad \quad T^{\perp} M=F \mathcal{D}^{\theta} \oplus \nu,
$$

where $\nu$ denotes the orthogonal complementary distribution of $F \mathcal{D}^{\theta}$ in $T^{\perp} M$.

Next, for an element $U \in T M$ in a semi-slant submanifold $M$, we write

$$
U=T_{1} U+T_{2} U
$$

where $T_{1} U$ (resp. $T_{2} U$ ) denotes the $\mathcal{D}\left(\right.$ resp. $\mathcal{D}^{\theta}$ ) component of $U$.

It follows from (2.3) and (2.4) that

$$
J U=J T_{1} U+T T_{2} U+F T_{2} U,
$$

where $J T_{1} U \in \mathcal{D}, T T_{2} U \in \mathcal{D}^{\theta}$ and $F T_{2} U \in F \mathcal{D}^{\theta} \subset T^{\perp} M$. Thus if we put

$$
P U=J T_{1} U+T T_{2} U
$$

for any $U \in T M$, then

$$
P^{2} U=-T_{1} U-T_{2} U-t F T_{2} U
$$

for any $U \in T M$.

Now we easily get from (2.7) the folowing statement:

Proposition 2.2. In a semi-slant submanifold of an almost Hermitian manifold $\tilde{M}$, the operator $P$ defined by (2.6) is an almost complex structure in the holomorphic distribution $\mathcal{D}$. 
The covariant differentiation $\bar{\nabla}$ of $T_{1}, T_{2}, T, F, t$ and $f$ are defined as follows:

$$
\begin{aligned}
\left(\bar{\nabla}_{U} T_{1}\right) V & =\nabla_{U}\left(T_{1} V\right)-T_{1} \nabla_{U} V, & & \left(\bar{\nabla}_{U} T_{2}\right) V=\nabla_{U}\left(T_{2} V\right)-T_{2} \nabla_{U} V, \\
\left(\bar{\nabla}_{U} T\right) V & =\nabla_{U}(T V)-T \nabla_{U} V, & \left(\bar{\nabla}_{U} F\right) V & =\nabla_{U}^{\perp}(F V)-F \nabla_{U} V, \\
\left(\bar{\nabla}_{U} t\right) \xi & =\nabla_{U}(t \xi)-t \nabla_{U}^{\perp} \xi, & \left(\bar{\nabla}_{U} f\right) \xi & =\nabla_{U}^{\perp}(f \xi)-f \nabla_{U}^{\perp} \xi
\end{aligned}
$$

where $U, V \in T M$ and $\xi \in T^{\perp} M$.

Moreover, if we define the covariant differentiation $\bar{\nabla}$ of $P$ by

$$
\left(\bar{\nabla}_{U} P\right) V=\nabla_{U}(P V)-P \nabla_{U} V
$$

then

$$
\begin{array}{r}
\left(\bar{\nabla}_{U} P\right) V=\left(\tilde{\nabla}_{U} J\right) T_{1} V+J\left(\bar{\nabla}_{U} T_{1}\right) V+\left(\bar{\nabla}_{U} T\right)\left(T_{2} V\right)+ \\
+T\left(\bar{\nabla}_{U} T_{2}\right) V+J \sigma\left(U, T_{1} V\right)-\sigma\left(U, J T_{1} V\right) .
\end{array}
$$

Write

$$
\left(\tilde{\nabla}_{U} J\right) V=\mathcal{P}_{U} V+\mathcal{Q}_{U} V
$$

where $\mathcal{P}_{U} V$ (resp. $\mathcal{Q}_{U} V$ ) denotes the tangential (resp. normal) part of $\left(\tilde{\nabla}_{U} J\right) V$.

V. A. Khan and M. A. Khan proved the following statement:

Proposition 2.3. [9]. The holomorphic distribution $\mathcal{D}$ on a semi-slant submanifold of an almost Hermitian manifold is integrable if and only if

$$
\mathcal{Q}_{X} Y-\mathcal{Q}_{Y} X=\sigma(X, T Y)-\sigma(Y, T X)
$$

for any $X, Y \in \mathcal{D}$. The slant distribution $\mathcal{D}^{\theta}$ on a semi-slant submanifold of an almost Hermitian manifold is integrable if and only if

$$
T_{1}\left(\nabla_{Z} T W-\nabla_{W} T Z+A_{F Z} W-A_{F W} Z+\mathcal{P}_{W} Z-\mathcal{P}_{Z} W\right)=0
$$

for any $Z, W \in \mathcal{D}^{\theta}$.

Using these proposition, we proved the following result:

Theorem 2.4. [11]. (I) The holomorphic distribution $\mathcal{D}$ of a semi-slant submanifold $M$ in an l.c.K.-manifold $\tilde{M}(J, \tilde{g}, \alpha)$ is integrable if and only if

$$
\sigma(X, T Y)-\sigma(Y, T X)=2 \tilde{g}(T X, Y) \alpha_{2}^{\sharp}
$$

(II) The slant distribution $\mathcal{D}^{\theta}$ of a semi-slant submanifold $M$ in an l,c,K.manifold $\tilde{M}(J, \tilde{g}, \alpha)$ is integrable if and only if

$$
\begin{aligned}
& T_{1}\left(\nabla_{Z} T W-\nabla_{W} T Z+A_{F Z} W-A_{F W} Z+\right. \\
& \left.+\tilde{g}\left(\alpha_{1}^{\sharp}, W\right) T Z-\tilde{g}\left(\alpha_{1}^{\sharp}, Z\right) T W+2 \tilde{g}(T W, Z) \alpha_{1}^{\sharp}\right)=0
\end{aligned}
$$


or equivalently

$$
\begin{aligned}
T_{1}\left\{\left(\bar{\nabla}_{Z} T\right) W-(\right. & \left.\bar{\nabla}_{W} T\right) Z+T[Z, W]+A_{F Z} W-A_{F W} Z+ \\
& \left.+\tilde{g}\left(\alpha_{1}^{\sharp}, W\right) T Z-\tilde{g}\left(\alpha_{1}^{\sharp}, Z\right) T W+2 \tilde{g}(T W, Z) \alpha_{1}^{\sharp}\right\}=0,
\end{aligned}
$$

where $Z, W \in \mathcal{D}^{\theta}$, and $[Z, W]$ is the Lie bracket of $Z$ and $W$.

\section{WARPED PRODUCT SEMI-SLANT SUBMANIFOLDS IN AN L.C.K.-MANIFOLD}

Let $M$ be a semi-slant submanifold of an almost Hermitian manifold $\tilde{M}(J, \tilde{g})$. Suppose that the distributions $\mathcal{D}$ and $\mathcal{D}^{\theta}$ are integrable, and let $M_{\mathcal{D}}$ (resp. $M_{\mathcal{D}^{\theta}}$ ) be the maximal integral submanifold of $\mathcal{D}$ (resp. $\mathcal{D}^{\theta}$ ). Then $M$ is a product manifold of $M_{\mathcal{D}}$ and $M_{\mathcal{D}^{\theta}}$, that is,

$$
M=M_{\mathcal{D}} \otimes M_{\mathcal{D}^{\theta}}
$$

Therefore we can write

$$
T \tilde{M}=T M_{\mathcal{D}} \oplus T M_{\mathcal{D}^{\theta}} \oplus F T M_{\mathcal{D}^{\theta}} \oplus \nu
$$

where $\nu$ is the complementaly orthogonal subbundle of $F T M_{\mathcal{D}^{\theta}}=F \mathcal{D}^{\theta}$ in $T^{\perp} M$. We will call the submanifold $M_{\mathcal{D}}$ (resp. $\left.M_{\mathcal{D}^{\theta}}\right)$ the holomorphic (resp. slant) component of $M$.

Given a differentiable function $f_{1}$ (resp. $f_{2}$ ) on $M_{\mathcal{D}^{\theta}}\left(\right.$ resp. $\left.M_{\mathcal{D}}\right)$ ), define the following warped product submanifolds

$$
M_{1}=M_{\mathcal{D}} \otimes_{f_{1}} M_{\mathcal{D}^{\theta}}, \quad \quad M_{2}=M_{\mathcal{D}^{\theta}} \otimes_{f_{2}} M_{\mathcal{D}}
$$

We say that $M_{1}$ (resp. $M_{2}$ ) is the first (resp. second) type warped product semi-slant submanifold of an almost Hermitian manifold.

In this paper, we mainly consider the first type warped product semislant submanifold in an l.c.K.-manifold.

Let $M$ be the first type warped product semi-slant submanifold in an l.c.K.-manifold $\tilde{M}$. Then the induced metric tensor $g$ in $M$ of $\tilde{M}$ is given by

$$
g(U, V)=e^{f^{2}} g_{\mathcal{D}}\left(\pi_{\mathcal{D}} * U, \pi_{\mathcal{D}} * V\right)+g_{\mathcal{D}^{\theta}}\left(\pi_{\mathcal{D}^{\theta}} * U, \pi_{\mathcal{D}^{\theta}} * V\right)
$$

for any $U, V \in T M$, where $g_{\mathcal{D}}$ (resp. $g_{\mathcal{D}^{\theta}}$ ) denotes the Riemannian metric on $M_{\mathcal{D}}$ (resp. $\left.M_{\mathcal{D}^{\theta}}\right), \pi_{\mathcal{D}}$ (resp. $\pi_{\mathcal{D}^{\theta}}$ ) is the projection operator of $M$ to $M_{\mathcal{D}}$ (resp. $M_{\mathcal{D}^{\theta}}$ ) and $f$ is a certain positive differentiable function on $M_{\mathcal{D}^{\theta}}$. 
Now, let $\tilde{\nabla}, \nabla, \nabla^{\mathcal{D}}$ and $\nabla^{\mathcal{D}^{\theta}}$ be the covariant differentiations with respect to $\tilde{g}, g, g_{\mathcal{D}}$ and $g_{\mathcal{D}^{\theta}}$, respectively. Then by (1.4)

$$
\begin{aligned}
\nabla_{X} Y & =\nabla^{\mathcal{D}}{ }_{X} Y-f^{2} e^{f^{2}}\left(\triangle_{1} \log f\right) g_{\mathcal{D}}(X, Y), \\
\nabla_{X} Z & =\nabla_{Z} X=f^{2}(Z \log f) X, \\
\nabla_{Z} W & =\nabla^{\mathcal{D}^{\theta}}{ }_{Z} W
\end{aligned}
$$

for any $X, Y \in \mathcal{D}$ and $Z, W \in \mathcal{D}^{\theta}$, where we put

$$
\triangle_{1} \log f=g_{\mathcal{D}^{\theta}}{ }^{c e}\left(\partial_{c} \log f\right) \partial_{e} .
$$

Let $M$ be a semi-slant submanifold with distributions $\mathcal{D}, \mathcal{D}^{\theta}$ be an almost Hermitian manifold $\tilde{M}$, $\operatorname{dim} \mathcal{D}=2 p, \operatorname{dim} \mathcal{D}^{\perp}=q$, and $\operatorname{dim} \nu=2 s$. Then we take the following generalized adopted frame in $\tilde{M}$ :

(1) $\left\{e_{1}, e_{2}, \ldots, e_{p}, e_{1}^{*}, e_{2}^{*}, \ldots, e_{p}^{*}\right\}$ is an orthonormal frame of $\mathcal{D}$, where $e_{i}^{*}=J e_{i}$ for $i \in\{1,2, \ldots, p\}$

(2) $\left\{e_{2 p+1}, e_{2 p+2}, \ldots, e_{2 p+q}\right\}$ is an orthonormal frame of $\mathcal{D}^{\theta}$ such that the vectors $F e_{2 p+1}, F e_{2 p+2}, \ldots, F e_{2 p+q}$ are orthogonal in $F \mathcal{D}^{\theta}$.

(3) $\left\{e_{n+q+1}, e_{n+q+2}, \ldots, e_{n+q+s}, e_{n+q+1}^{*}, e_{n+q+2}^{*}, \ldots, e_{n+q+s}^{*}\right\}$ is an orthonormal frame of $\nu$, where $e_{n+q+a}^{*}=J e_{n+q+a}$ for $a \in\{1,2, \ldots, s\}$;

(4) $e_{2 p+a}^{*}=\frac{F e_{2 p+a}}{\left\|F e_{2 p+a}\right\|}$ for $a \in\{1,2, \ldots, q\}$.

Hereafter, for a tensor field $T$ of $(0, s)$-type, we write $T_{\mu_{1}, \mu_{2}, \ldots, \mu_{s}}$ instead of $T\left(e_{\mu_{1}}, e_{\mu_{2}}, \ldots, e_{\mu_{s}}\right)$ with respect to the generalized adapted frame.

In the last paper $([11])$, using the Gauss equation, we proved

Proposition 3.1. In a first type warped product semi-slant submanifold in an l.c.K.-space form, the mean curvature $\|H\|$ satisfies the inequality

$$
\begin{gathered}
4 n\|H\|^{2}+8 p f^{2}\left\{(2 p-1) f^{2}\|\log f\|^{2}+2\left(2+f^{2}\right) \sum_{a=2 p+1}^{2 p+q}\left(e_{a} \log f\right)^{2}\right\}+ \\
+\left(n^{2}+2 n-3 q\right) c+3 c \sum_{b, a=2 p+1}^{2 p+q}\left\{T_{b a}\right\}^{2}-4\left(e^{f^{2}} \tau^{\mathcal{D}}+\tau^{\mathcal{D}^{\theta}}\right)+ \\
+16 p f^{2} \sum_{b, a=2 p+1}^{2 p+q} \nabla^{\mathcal{D}^{\theta}} e_{a} \nabla^{\mathcal{D}^{\theta}} e_{a} \log f+6(n-2) \sum_{\mu=1}^{n} P_{\mu \mu}+ \\
+6 \sum_{a=2 p+1}^{2 p+q} P_{a a}-6 \sum_{b, a=2 p+1}^{2 p+q} P\left(J e_{b}, e_{a}\right) T_{b a} \geq 0 .
\end{gathered}
$$


In particular, the equality case of (3.6) is that our submanifold is totally geodesic. Then we have the following equation for the warping function $f$

$$
\begin{aligned}
8 p f^{2}\left\{(2 p-1) f^{2}\|\log f\|^{2}+2\left(2+f^{2}\right) \sum_{a=2 p+1}^{2 p+q}\left(e_{a} \log f\right)^{2}\right\}+ \\
+\left(n^{2}+2 n-3 q\right) c+3 c \sum_{b, a=2 p+1}^{2 p+q}\left\{T_{b a}\right\}^{2}-4\left(e^{f^{2}} \tau^{\mathcal{D}}+\tau^{\mathcal{D}^{\theta}}\right)+ \\
+16 p f^{2} \sum_{b, a=2 p+1}^{2 p+q} \nabla^{\mathcal{D}^{\theta}} e_{a} \nabla^{\mathcal{D}^{\theta}} e_{a} \log f+6(n-2) \sum_{\mu=1}^{n} P_{\mu \mu}+ \\
+6 \sum_{a=2 p+1}^{2 p+q} P_{a a}-6 \sum_{b, a=2 p+1}^{2 p+q} P\left(J e_{b}, e_{a}\right) T_{b a}=0 .
\end{aligned}
$$

From which, we obtain

$$
\begin{gathered}
\left(n^{2}+2 n-3 q\right) c+3 c \sum_{b, a=2 p+1}^{2 p+q}\left\{T_{b a}\right\}^{2}-4\left(e^{f^{2}} \tau^{\mathcal{D}}+\tau^{\mathcal{D}^{\theta}}\right)+ \\
+16 p f^{2} \sum_{b, a=2 p+1}^{2 p+q} \nabla^{\mathcal{D}^{\theta}} e_{a} \nabla^{\mathcal{D}^{\theta}} e_{a} \log f+6(n-2) \sum_{\mu=1}^{n} P_{\mu \mu}+ \\
+6 \sum_{a=2 p+1}^{2 p+q} P_{a a}-6 \sum_{b, a=2 p+1}^{2 p+q} P\left(J e_{b}, e_{a}\right) T_{b a} \leq 0 .
\end{gathered}
$$

\section{Semi-Slant Submanifolds With the Parallel SECOND} FUNDAMENTAL FORM

Using (1.1), the curvature tensor $\tilde{R}$ of the first type warped product semi-slant submanifold $M$ in an l.c.K.-space form $\tilde{M}(c)$ is separated, with respect to the generalized adapted frame, as

$$
\begin{aligned}
4 \tilde{R}_{j i h a^{*}} & =3\left(P_{j a^{*}} \delta_{i h}-P_{i a^{*}} \delta_{j h}\right), \\
4 \tilde{R}_{j i h^{*} a^{*}} & =-P_{j^{*} a^{*}} \delta_{i h}+P_{i^{*} a^{*}} \delta_{j h}, \\
4 \tilde{R}_{j i^{*} h a^{*}} & =-3 P_{i^{*} a^{*}} \delta_{j h}+P_{j^{*} a^{*}} \delta_{i h}+2 P_{h^{*} a^{*}} \delta_{j i}, \\
4 \tilde{R}_{j i^{*} h^{*} a^{*}} & =3 P_{j a^{*}} \delta_{i h}-P_{i a^{*}} \delta_{j h}-2 P_{h a^{*}} \delta_{j i}, \\
4 \tilde{R}_{j^{*} i^{*} h^{*} a^{*}} & =3\left(P_{j^{*} a^{*}} \delta_{i h}-P_{i^{*} a^{*}} \delta_{j h}\right), \\
2 \tilde{R}_{j i b a^{*}} & =P_{j^{*} i} F_{b a},
\end{aligned}
$$


Warped product semi-slant submanifolds, II

$$
\begin{aligned}
& 2 \tilde{R}_{j i^{*} b a^{*}}=-c \delta_{j i} F_{b a}+P_{j i} F_{b a}+\left(T_{b}^{c} P_{c a^{*}}+F_{b}^{c} P_{c^{*} a^{*}}\right) \delta_{j i}, \\
& 2 \tilde{R}_{j^{*} i^{*} b a^{*}}=-P_{j i^{*}} F_{b a}, \\
& 4 \tilde{R}_{j c b a^{*}}=3 P_{j a^{*}} \delta_{c b}-P_{j^{*} a^{*}} T_{c b}+P_{j^{*} b} F_{c a}+2 P_{j^{*} c} F_{b a}, \\
& 4 \tilde{R}_{j^{*} c b a^{*}}=3 P_{j^{*} a^{*}} \delta_{c b}+P_{j a^{*}} T_{c b}-P_{j b} F_{c a}-2 P_{j c} F_{b a}, \\
& 4 \tilde{R}_{d c b a^{*}}=c\left(F_{d a} T_{c b}-T_{d b} F_{c a}-2 T_{d c} F_{b a}\right)+3\left(P_{d a^{*}} \delta_{c b}-P_{c a^{*}} \delta_{d b}\right)- \\
& -\left(T_{d}^{c} P_{c a^{*}}+F_{d}^{c} P_{c^{*} a^{*}}\right) T_{c b}+\left(T_{d}^{e} P_{e b}+F_{d}^{e} P_{e^{*} b}\right) F_{c a}- \\
& -\left(T_{c}{ }^{e} P_{e b}+F_{c}{ }^{e} P_{e^{*} b}\right) F_{d a}+\left(T_{c}^{e} P_{e a^{*}}+F_{c}{ }^{e} P_{e^{*} a^{*}}\right) T_{d b}+ \\
& +2\left\{\left(T_{d}^{e} P_{e c}+F_{d}^{e} P_{e^{*} c}\right) F_{b a}+\left(T_{b}^{e} P_{e a^{*}}+F_{b}{ }^{e} P_{e^{*} a^{*}}\right) T_{d c}\right\}, \\
& 4 \tilde{R}_{j i h r}=3\left(P_{j r} \delta_{i h}-P_{i r} \delta_{j h}\right), \\
& 4 \tilde{R}_{j i h^{*} r}=-P_{j^{*} r} \delta_{i h}+P_{i^{*} r} \delta_{j h}, \\
& 4 \tilde{R}_{j i^{*} h r}=-3 P_{i^{*} r} \delta_{j h}+P_{j^{*} r} \delta_{i h}+2 P_{h^{*} r} \delta_{j i}, \\
& 4 \tilde{R}_{j i^{*} h^{*} r}=3 P_{j r} \delta_{i h}-P_{i r} \delta_{j h}-2 P_{h r} \delta_{j i}, \\
& 4 \tilde{R}_{j^{*} i^{*} h r}=-P_{j r} \delta_{i h}+P_{i r} \delta_{j h}, \\
& 4 \tilde{R}_{j^{*} i^{*} h^{*} r}=3\left(P_{j^{*} r} \delta_{i h}-P_{i^{*} r} \delta_{j h}\right), \\
& \tilde{R}_{\text {jiar }}=0 \text {, } \\
& 2 \tilde{R}_{j i^{*} a r}=\left(T_{a}{ }^{e} P_{e r}+F_{a}{ }^{e} p_{e^{*} r}\right) \delta_{j i}, \\
& \tilde{R}_{j^{*} i^{*} a r}=0, \\
& 4 \tilde{R}_{j b a r}=3 P_{j r} \delta_{b a}-P_{j^{*} r} T_{b a}, \\
& 4 \tilde{R}_{j^{*} b a r}=3 P_{j^{*} r} \delta_{b a}+P_{j r} T_{b a}, \\
& 4 \tilde{R}_{c b a r}=3\left(P_{c r} \delta_{b a}-P_{b r} \delta_{c a}\right)-\left(T_{c}^{e} P_{e r}+F_{c}^{e} P_{c}^{e} P_{e^{*} r}\right) T_{b a} \\
& +\left(T_{b}{ }^{e} P_{e r}+F_{b}{ }^{e} P_{e^{*} r}\right) T_{c a}+2\left(T_{a}{ }^{e} P_{e r}+F_{a}{ }^{e} P_{e^{*} r}\right) T_{c b}
\end{aligned}
$$

for any $j, i, h \in\{1,2, \ldots, p\}, d, c, b, a \in\{2 p+1,2 p+2, \ldots, 2 p+q\}$ and $r \in\{n+q+1, n+q+2, \ldots, m\}$.

Thus, by virtue of previous formulas the Codazzi equation (2.1) is separated as

$$
\begin{aligned}
4\left\{\tilde{g}\left(\bar{\nabla}_{j} \sigma_{i h}, e_{a}{ }^{*}\right)-\tilde{g}\left(\bar{\nabla}_{i} \sigma_{j h}, e_{a}{ }^{*}\right)\right\} & =3\left(P_{j a^{*}} \delta_{i h}-P_{i a^{*}} \delta_{j h}\right), \\
4\left\{\tilde{g}\left(\bar{\nabla}_{j} \sigma_{i h^{*}}, e_{a}{ }^{*}\right)-\tilde{g}\left(\bar{\nabla}_{i} \sigma_{j h^{*}}, e_{a}{ }^{*}\right)\right\} & =-P_{j^{*} a^{*}} \delta_{i h}+P_{i^{*} a^{*}} \delta_{j h}, \\
4\left\{\tilde{g}\left(\bar{\nabla}_{j} \sigma_{i^{*} h}, e_{a}{ }^{*}\right)-\tilde{g}\left(\bar{\nabla}_{i^{*}} \sigma_{j h}, e_{a}{ }^{*}\right)\right\} & =-3 P_{i^{*} a^{*}} \delta_{j h}+P_{j^{*} a^{*}} \delta_{i h}+2 P_{h^{*} a^{*}} \delta_{j i}, \\
4\left\{\tilde{g}\left(\bar{\nabla}_{j} \sigma_{i^{*} h^{*}}, e_{a}{ }^{*}\right)-\tilde{g}\left(\bar{\nabla}_{i^{*}} \sigma_{j h^{*}}, e_{a}{ }^{*}\right)\right\} & =3 P_{j a^{*}} \delta_{i h}-P_{i a^{*}} \delta_{j h}-2 P_{h a^{*}} \delta_{j i}, \\
4\left\{\tilde{g}\left(\bar{\nabla}_{j^{*}} \sigma_{i^{*} h^{*}}, e_{a}{ }^{*}\right)-\tilde{g}\left(\bar{\nabla}_{i^{*}} \sigma_{j^{*} h^{*}}, e_{a}{ }^{*}\right)\right\} & =3\left(P_{j^{*} a^{*}} \delta_{i h}-P_{i^{*} a^{*}} \delta_{j h}\right),
\end{aligned}
$$




$$
\begin{aligned}
& 2\left\{\tilde{g}\left(\bar{\nabla}_{j} \sigma_{i b}, e_{a}^{*}\right)-\tilde{g}\left(\bar{\nabla}_{i} \sigma_{j b}, e_{a}{ }^{*}\right)\right\}=P_{j^{*} i} F_{b a} \\
& 2\left\{\tilde{g}\left(\bar{\nabla}_{j} \sigma_{i^{*} b}, e_{a}^{*}\right)-\tilde{g}\left(\bar{\nabla}_{i^{*}} \sigma_{j b}, e_{a}{ }^{*}\right)\right\}=-c \delta_{j i} F_{b a}+P_{j i} F_{b a}+ \\
& +\left\{T_{b}^{c} P_{c a^{*}}+F_{b}^{c} P_{c^{*} a^{*}}\right\} \delta_{j i}, \\
& 2\left\{\tilde{g}\left(\bar{\nabla}_{j^{*}} \sigma_{i^{*} b}, e_{a}{ }^{*}\right)-\tilde{g}\left(\bar{\nabla}_{i^{*}} \sigma_{j^{*} b}, e_{a}{ }^{*}\right)\right\}=-P_{j i^{*}} F_{b a}, \\
& 4\left\{\tilde{g}\left(\bar{\nabla}_{j} \sigma_{c b}, e_{a}{ }^{*}\right)-\tilde{g}\left(\bar{\nabla}_{c} \sigma_{j b}, e_{a}{ }^{*}\right)\right\}=3 P_{j a^{*}} \delta_{c b}-P_{j^{*} a^{*}} T_{c b}+ \\
& +P_{j^{*} b} F_{c a}+2 P_{j^{*} c} F_{b a}, \\
& 4\left\{\tilde{g}\left(\bar{\nabla}_{j^{*}} \sigma_{c b}, e_{a}{ }^{*}\right)-\tilde{g}\left(\bar{\nabla}_{c} \sigma_{j^{*} b}, e_{a}{ }^{*}\right)\right\}=3 P_{j^{*} a^{*}} \delta_{c b}+P_{j a^{*}} T_{c b}- \\
& -P_{j b} F_{c a}-2 P_{j c} F_{b a} \\
& 4\left\{\tilde{g}\left(\bar{\nabla}_{d} \sigma_{c b}, e_{a}{ }^{*}\right)-\tilde{g}\left(\bar{\nabla}_{c} \sigma_{d b}, e_{a}{ }^{*}\right)\right\}=c\left(F_{d a} T_{c b}-T_{d b} F_{c a}-2 T_{d c} F_{b a}\right) \\
& +3\left(P_{d a^{*}} \delta_{c b}-P_{c a^{*}} \delta_{d b}\right)-\left(T_{d}^{e} P_{e a^{*}}+F_{d}{ }^{e} P_{e^{*} a^{*}}\right) T_{c b} \\
& +\left(T_{d}^{e} P_{e b}+F_{d}^{e} P_{b e^{*}}\right) F_{c a}-\left(T_{c}^{e} P_{e b}+F_{c}{ }^{e} P_{b c^{*}}\right) F_{d a} \\
& +\left(T_{c}{ }^{e} P_{e a^{*}}+F_{c}{ }^{e} P_{e^{*} a^{*}}\right) T_{d b}+2\left\{\left(T_{d}^{e} P_{e c}+F_{d}{ }^{c} P_{c e^{*}}\right) F_{b a}\right. \\
& \left.+\left(T_{b}^{e} P_{e a^{*}}+F_{b}^{e} P_{e^{*} a^{*}}\right) T_{d c}\right\}, \\
& 4\left\{\tilde{g}\left(\bar{\nabla}_{j} \sigma_{i h}, e_{r}\right)-\tilde{g}\left(\bar{\nabla}_{i} \sigma_{j h}, e_{r}\right)\right\}=3\left(P_{j r} \delta_{i h}-P_{i r} \delta_{j h}\right), \\
& 4\left\{\tilde{g}\left(\bar{\nabla}_{j} \sigma_{i h^{*}}, e_{r}\right)-\tilde{g}\left(\bar{\nabla}_{i} \sigma_{j h^{*}}, e_{r}\right)\right\}=-P_{j^{*} r} \delta_{i h}+P_{i^{*} r} \delta_{j h}, \\
& 4\left\{\tilde{g}\left(\bar{\nabla}_{j} \sigma_{i^{*} h}, e_{r}\right)-\tilde{g}\left(\bar{\nabla}_{i^{*}} \sigma_{j h}, e_{r}\right)\right\}=-3 P_{i^{*} r} \delta_{j h}+P_{j^{*} r} \delta_{i h}+2 P_{h^{*} r} \delta_{j i}, \\
& 4\left\{\tilde{g}\left(\bar{\nabla}_{j} \sigma_{i^{*} h^{*}}, e_{r}\right)-\tilde{g}\left(\bar{\nabla}_{i^{*}} \sigma_{j h^{*}}, e_{r}\right)\right\}=3 P_{j r} \delta_{i h}-P_{i r} \delta_{j h}-2 P_{h r} \delta_{j i}, \\
& 4\left\{\tilde{g}\left(\bar{\nabla}_{j^{*}} \sigma_{i^{*} h}, e_{r}\right)-\tilde{g}\left(\bar{\nabla}_{i^{*}} \sigma_{j^{*} h}, e_{r}\right)\right\}=-P_{j r} \delta_{i h}+P_{i r} \delta_{j h}, \\
& 4\left\{\tilde{g}\left(\bar{\nabla}_{j^{*}} \sigma_{i^{*} h^{*}}, e_{r}\right)-\tilde{g}\left(\bar{\nabla}_{i^{*}} \sigma_{j^{*} h^{*}}, e_{r}\right)\right\}=3\left(P_{j^{*} r} \delta_{i h}-P_{i^{*} r} \delta_{j h}\right), \\
& \tilde{g}\left(\bar{\nabla}_{j} \sigma_{i a}, e_{r}\right)-\tilde{g}\left(\bar{\nabla}_{i} \sigma_{j a}, e_{r}\right)=0, \\
& 4\left\{\tilde{g}\left(\bar{\nabla}_{j} \sigma_{i^{*} a}, e_{r}\right)-\tilde{g}\left(\bar{\nabla}_{i^{*}} \sigma_{j a}, e_{r}\right)\right\}=\left(T_{a}{ }^{c} P_{c r}+F_{a}{ }^{c} P_{c^{*} r}\right) \delta_{j i}, \\
& \tilde{g}\left(\bar{\nabla}_{j^{*}} \sigma_{i^{*} a}, e_{r}\right)-\tilde{g}\left(\bar{\nabla}_{i^{*}} \sigma_{j^{*} a}, e_{r}\right)=0, \\
& 4\left\{\tilde{g}\left(\bar{\nabla}_{j} \sigma_{b a}, e_{r}\right)-\tilde{g}\left(\bar{\nabla}_{b} \sigma_{j a}, e_{r}\right)\right\}=3 P_{j r} \delta_{b a}-P_{j^{*} r} T_{b a}, \\
& 4\left\{\tilde{g}\left(\bar{\nabla}_{j^{*}} \sigma_{b a}, e_{r}\right)-\tilde{g}\left(\bar{\nabla}_{b^{*}} \sigma_{j a}, e_{r}\right)\right\}=3 P_{j^{*} r} \delta_{b a}+P_{j r} T_{b a}, \\
& 4\left\{\tilde{g}\left(\bar{\nabla}_{c} \sigma_{b a}, e_{r}\right)-\tilde{g}\left(\bar{\nabla}_{b} \sigma_{c a}, e_{r}\right)\right\}=3\left(P_{c r} \delta_{b a}-P_{b r} \delta_{c a}\right)- \\
& -\left(T_{c}{ }^{e} P_{e r}+F_{c}{ }^{e} P_{e^{*} r}\right) T_{b a}+\left(T_{b}^{e} P_{e r}+F_{b}{ }^{e} P_{e^{*} r}\right) T_{c a}+ \\
& +2\left(T_{a}{ }^{e} P_{e r}+F_{a}{ }^{e} P_{e^{*} r}\right) T_{c b} \text {, }
\end{aligned}
$$

for all $j, i, h \in\{1,2, \ldots, p\}, d, c, b, a \in\{2 p+1,2 p+2, \ldots, 2 p+q\}$ and $r \in\{n+q+1, n+q+2, \ldots, m\}$.

Now we assume that the second fundamental form $\sigma$ is parallel, that is, $\bar{\nabla} \sigma=0$. Then we have from the above formulae that the tensor field $P_{B A}$ 
is written as

$$
\begin{aligned}
\left(P_{B A}\right) & =\left(\begin{array}{cccccc}
P_{j i} & P_{j i^{*}} & P_{j a} & P_{j a^{*}} & P_{j r} & P_{j r^{*}} \\
P_{j^{*} i} & P_{j^{*} i^{*}} & P_{j^{*} a} & P_{j^{*} a^{*}} & P_{j^{*} r} & P_{j^{*} r^{*}} \\
P_{b i} & P_{b i^{*}} & P_{b a} & P_{b a^{*}} & P_{b r} & P_{b r^{*}} \\
P_{b^{*} i} & P_{b^{*} i^{*}} & P_{b^{*} a} & P_{b^{*} a^{*}} & P_{b^{*} r} & P_{b^{*} r^{*}} \\
P_{r i} & P_{r i^{*}} & P_{r a} & P_{r a^{*}} & P_{s r} & P_{s r^{*}} \\
P_{s^{*} i} & P_{s^{*} i^{*}} & P_{s^{*} a} & P_{s^{*} a^{*}} & P_{s^{*} r} & P_{s^{*} r^{*}}
\end{array}\right) \\
& =\left(\begin{array}{cccccc}
\alpha \delta_{j i} & 0 & 0 & 0 & 0 & 0 \\
0 & \alpha \delta_{j i} & 0 & 0 & 0 & 0 \\
0 & 0 & P_{b a} & 0 & 0 & 0 \\
0 & 0 & 0 & P_{b^{*} a^{*}} & P_{b^{*} r} & P_{b^{*} r^{*}} \\
0 & 0 & 0 & P_{s a^{*}} & P_{s r} & P_{s r^{*}} \\
0 & 0 & 0 & P_{s^{*} a^{*}} & P_{s * r} & P_{s r}
\end{array}\right) .
\end{aligned}
$$

for $p, q \geq 1$ and a certain function $\alpha$. Thus we have

Theorem 4.1. In the first type warped product semi-slant submanifold $M$ with parallel second fundamental form $\sigma$ of an l.c.K.-space form $\tilde{M}(c)$, if $p$ and $q$ are bigger than 1, then the tensor field $P_{B A}$ is written by (4.1).

\section{Normally flat WARPED PROduCt SEMI-SLANT SUBMANIFOLdS}

Finally, we calculate the Ricci equation in a warped product semi-slant submanifold in an l.c.K.-space form.

By virtue of (1.1), we can separate the Riemannian curvature tensor $\tilde{R}(U, V, W, Z)$ for $U, V, W, Z \in \tilde{T} M$ as

$$
\begin{gathered}
2 \tilde{R}\left(X_{1}, X_{2}, F Z_{1}, F Z_{2}\right)=-c \tilde{g}\left(T X_{1}, X_{2}\right) \tilde{g}\left(J F Z_{1}, Z_{2}\right) \\
+P\left(J X_{1}, X_{2}\right) \tilde{g}\left(J F Z_{1}, F Z_{2}\right)+P\left(J F Z_{1}, F Z_{2}\right) \tilde{g}\left(J X_{1}, X_{2}\right), \\
2 \tilde{R}\left(X_{1}, X_{2}, F Z_{1}, \xi\right)=P\left(J F Z_{1}, \xi_{1}\right) \tilde{g}\left(J X_{1}, X_{2}\right), \\
2 \tilde{R}\left(X_{1} \cdot X_{2}, \xi_{1}, \xi_{2}\right)=-c \tilde{g}\left(J X_{1}, X_{2}\right) \tilde{g}\left(J \xi_{1}, \xi_{2}\right) \\
\quad+P\left(J X_{1}, X_{2}\right) \tilde{b}\left(J \xi_{1}, \xi_{2}\right)+P\left(J \xi_{1}, \xi_{2}\right) \tilde{g}\left(J X_{1}, X_{2}\right), \\
4 \tilde{R}\left(X_{1}, Z_{1}, F Z_{2}, F Z_{3}\right)=-P\left(J X_{1}, F Z_{3}\right) \tilde{g}\left(F Z_{1}, F Z_{2}\right) \\
\quad+P\left(J X_{1}, F Z_{2}\right) \tilde{g}\left(F Z_{1}, F Z_{3}\right)+2 P\left(J X_{1}, Z_{1}\right) \tilde{g}\left(J F Z_{2}, F Z_{3}\right), \\
4 \tilde{R}\left(X_{1}, Z_{1}, F Z_{2}, \xi_{1}\right)=-P\left(J X_{1}, \xi_{1}\right) \tilde{g}\left(F Z_{1}, F Z_{2}\right), \\
2 \tilde{R}\left(X_{1}, Z_{1}, \xi_{1}, \xi_{2}\right)=P\left(J X_{1}, Z_{1}\right) \tilde{g}\left(J \xi_{1}, \xi_{2}\right), \\
4 \tilde{R}\left(Z_{1}, Z_{2}, F Z_{3}, F Z_{4}\right)=c\left\{\tilde{g}\left(F Z_{1}, F Z_{4}\right) \tilde{g}\left(F Z_{1}, F Z_{3}\right)\right. \\
\left.-\tilde{g}\left(F Z_{1}, F Z_{4}\right) \tilde{g}\left(F Z_{1}, F Z_{3}\right)-2 \tilde{g}\left(T Z_{1}, Z_{2}\right) \tilde{g}\left(J F Z_{3}, F Z_{4}\right)\right\} \\
-P\left(J Z_{1}, F Z_{4}\right) \tilde{g}\left(F Z_{2}, F Z_{3}\right)+P\left(J Z_{1}, F Z_{3}\right) \tilde{g}\left(F Z_{2}, F Z_{4}\right)
\end{gathered}
$$




$$
\begin{aligned}
& -P\left(J Z_{2}, F Z_{3}\right) \tilde{g}\left(F Z_{1}, F Z_{4}\right)+P\left(J Z_{2}, F Z_{4}\right) \tilde{g}\left(F Z_{1}, F Z_{3}\right) \\
& +2\left\{P\left(J Z_{1}, Z_{2}\right) \tilde{g}\left(J F Z_{3}, F Z_{4}\right)+P\left(J F Z_{3}, F Z_{4}\right) \tilde{g}\left(J Z_{1}, Z_{2}\right)\right\} \\
4 \tilde{R}\left(Z_{1},\right. & \left.Z_{2}, F Z_{3}, \xi_{1}\right)=-P\left(J Z_{1}, \xi\right) \tilde{g}\left(F Z_{2}, F Z_{3}\right) \\
& +P\left(J Z_{2}, \xi_{1}\right) \tilde{g}\left(F Z_{1}, F Z_{3}\right)+2 P\left(J F Z_{3}, \xi_{1}\right) \tilde{g}\left(J Z_{1}, Z_{2}\right), \\
2 \tilde{R}\left(Z_{1}, Z_{2}, \xi_{1}, \xi_{2}\right)=-c \tilde{g}\left(J Z_{1}, Z_{2}\right) \tilde{g}\left(J \xi_{1}, \xi_{2}\right) & +P\left(J Z_{1}, Z_{2}\right) \tilde{g}\left(J \xi_{1}, \xi_{2}\right)+P\left(J \xi_{1}, \xi_{2}\right) \tilde{g}\left(J Z_{1}, Z_{2}\right) \\
& +
\end{aligned}
$$

for any $X_{1}, X_{2} \in \mathcal{D}, Z_{1}, Z_{2}, Z_{3}, Z_{4} \in \mathcal{D}^{\theta}$ and $\xi_{1}, \xi_{2} \in \nu$. Hence we get from (2.2) that the Ricci equation is separated by

$$
\begin{aligned}
& 2 R^{\perp}\left(X_{1}, X_{2}, F Z_{1}, F Z_{2}\right)-2 \tilde{g}\left(\left[A_{F Z_{1}}, A_{F Z_{2}}\right] X_{1}, X_{2}\right)= \\
& -c \tilde{g}\left(J X_{1}, X_{2}\right) \tilde{g}\left(J F Z_{1}, F Z_{2}\right)+P\left(J X_{1}, X_{2}\right) \tilde{g}\left(J F Z_{1}, F Z_{2}\right) \\
& +P\left(J F Z_{1}, F Z_{2}\right) \tilde{g}\left(J X_{1}, X_{2}\right) \text {, } \\
& 2 R^{\perp}\left(X_{1}, X_{2}, F Z_{1}, \xi_{1}\right)-2 \tilde{g}\left(\left[A_{F Z_{1}}, A_{\xi_{1}}\right] X_{1}, X_{2}\right)=P\left(J F Z_{1}, \xi\right) \tilde{g}\left(J X_{1}, X_{2}\right), \\
& 2 R^{\perp}\left(X_{1}, X_{2}, \xi_{1}, \xi_{2}\right)-2 \tilde{g}\left(\left[A_{\xi_{1}}, A_{\xi_{2}}\right] X_{1}, X_{2}\right)=-c \tilde{g}\left(J X_{1}, X_{2}\right) \tilde{g}\left(J \xi_{1}, \xi_{2}\right)+ \\
& +P\left(J X_{1}, X_{2}\right) \tilde{g}\left(J \xi_{1}, \xi_{2}\right)+P\left(J \xi_{1}, \xi_{2}\right) \tilde{g}\left(J X_{1}, X_{2}\right), \\
& 4 R^{\perp}\left(X_{1}, Z_{1}, F Z_{2}, F Z_{3}\right)-4 \tilde{g}\left(\left[A_{F Z_{2}}, A_{F Z_{3}}\right] X_{1}, Z_{2}\right)= \\
& =-P\left(J X_{1}, F Z_{3}\right) \tilde{g}\left(F Z_{1}, F Z_{2}\right)+P\left(J X_{1}, F Z_{2}\right) \tilde{g}\left(F Z_{1}, F Z_{3}\right)+ \\
& +2 P\left(J X_{1}, Z_{1}\right) \tilde{g}\left(J F Z_{2}, F Z_{3}\right), \\
& 4 R^{\perp}\left(X_{1}, Z_{1}, F Z_{2}, \xi\right)-4 \tilde{g}\left(\left[A_{F Z_{2}}, A_{\xi_{1}}\right] X_{1}, Z_{1}\right)=-P\left(J X_{1}, \xi_{1}\right) \tilde{g}\left(F Z_{1}, F Z_{2}\right), \\
& 2 R^{\perp}\left(X_{1}, Z_{1}, \xi_{1}, \xi_{2}\right)-2 \tilde{g}\left(\left[A_{\xi_{1}}, A_{\xi_{2}}\right] X_{1}, Z_{1}\right)=P\left(J X_{1}, Z_{1}\right) \tilde{g}\left(J \xi_{1}, \xi_{2}\right), \\
& 4 R^{\perp}\left(Z_{1}, Z_{2}, F Z_{3}, F Z_{4}\right)-4 \tilde{g}\left(\left[A_{F Z_{3}}, A_{F Z_{4}}\right] Z_{1}, Z_{2}\right)= \\
& =c\left\{\tilde{g}\left(F Z_{1}, F Z_{4}\right) \tilde{g}\left(F Z_{2}, F Z_{3}\right)-\tilde{g}\left(F Z_{1}, F Z_{3}\right) \tilde{g}\left(F Z_{2}, F Z_{4}\right)-\right. \\
& \left.-2 \tilde{g}\left(T Z_{1}, Z_{2}\right) \tilde{g}\left(J F Z_{3}, F Z_{4}\right)\right\}-P\left(J Z_{1}, F Z_{4}\right) \tilde{g}\left(F Z_{2}, F Z_{3}\right)+ \\
& +P\left(J Z_{1}, F Z_{3}\right) \tilde{g}\left(F Z_{2}, F Z_{4}\right)-P\left(J Z_{2}, F Z_{3}\right) \tilde{g}\left(F Z_{1}, F Z_{4}\right)+ \\
& +P\left(J Z_{2}, F Z_{4}\right) \tilde{g}\left(F Z_{1}, F Z_{3}\right)+2\left\{P\left(J Z_{1}, Z_{2}\right) \tilde{g}\left(J F Z_{3}, F Z_{4}\right)+\right. \\
& \left.+P\left(J F Z_{3}, F Z_{4}\right) \tilde{g}\left(T Z_{1}, Z_{2}\right)\right\}, \\
& 4 R^{\perp}\left(Z_{1}, Z_{2}, F Z_{3}, \xi_{1}\right)-4 \tilde{g}\left(\left[A_{F Z_{3}}, A_{\xi_{1}}\right] Z_{1}, Z_{2}\right)=2 P\left(J F Z_{3}, \xi_{1}\right) \tilde{g}\left(T Z_{1}, Z_{2}\right)-
\end{aligned}
$$$$
-P\left(J Z_{1}, \xi_{1}\right) \tilde{g}\left(F Z_{2}, F Z_{3}\right)+P\left(J Z_{2}, \xi\right) \tilde{g}\left(F Z_{1}, F Z_{3}\right),
$$$$
4 R^{\perp}\left(Z_{1}, Z_{2}, \xi_{1}, \xi_{2}\right)-2 \tilde{g}\left(\left[A_{\xi_{1}}, A_{\xi_{2}}\right] Z_{1}, Z_{2}\right)=-c \tilde{g}\left(J Z_{1}, Z_{2}\right) \tilde{g}\left(J \xi_{1}, \xi_{2}\right)+
$$$$
+P\left(J Z_{1}, Z_{2}\right) \tilde{g}\left(J \xi_{1}, \xi_{2}\right)+P\left(J \xi_{1}, \xi_{2}\right) \tilde{g}\left(J Z_{1}, Z_{2}\right)
$$

for any $X_{1}, X_{2} \in \mathcal{D}, Z_{1}, Z_{2}, Z_{3}, Z_{4} \in \mathcal{D}^{\theta}$ and $\xi_{1}, \xi_{2} \in \nu$. 
Using the above equation, we have, for a generalized adopted frame, the following:

$$
\begin{aligned}
& 2 R^{\perp}{ }_{j i b^{*} a^{*}}-2 \tilde{g}\left(\left[A_{b^{*}}, A_{a^{*}}\right] e_{j}, e_{i}\right)=-c \tilde{g}\left(J e_{j}, e_{i}\right) \tilde{g}\left(J e_{b}{ }^{*}, e_{a}{ }^{*}\right)+ \\
& +P\left(J e_{j}, e_{i}\right) \tilde{g}\left(J e_{b}{ }^{*}, e_{a}{ }^{*}\right)+P\left(J e_{b}{ }^{*}, e_{a}{ }^{*}\right) \tilde{g}\left(J e_{j}, e_{i}\right), \\
& 2 R^{\perp}{ }_{j i a^{*} r}-2 \tilde{g}\left(\left[A_{a^{*}}, A_{r}\right] e_{j}, e_{i}\right)=P\left(J e_{a}{ }^{*}, e_{r}\right) \tilde{g}\left(J e_{j}, e_{i}\right), \\
& 2 R^{\perp}{ }_{j i s r}-2 \tilde{g}\left(\left[A_{e_{s}}, A_{e_{r}}\right] e_{j}, e_{i}\right)=-c \tilde{g}\left(J e_{j}, e_{i}\right) \tilde{g}\left(J e_{s}, e_{r}\right)+ \\
& +P\left(J e_{j}, e_{i}\right) \tilde{g}\left(J e_{s}, e_{r}\right)+P\left(J e_{s}, e_{r}\right) \tilde{g}\left(J e_{j}, e_{i}\right), \\
& 4 R^{\perp}{ }_{i c b^{*} a^{*}}-4 \tilde{g}\left(\left[A_{b^{*}}, A_{a^{*}}\right] e_{i}, e_{c}\right)=\left\|F e_{c}\right\|\left\{-P\left(J e_{i}, e_{a}{ }^{*}\right) \delta_{c b}+\right. \\
& \left.+P\left(J e_{i}, e_{b}{ }^{*}\right) \delta_{c a}\right\}+2 P\left(J e_{i}, e_{c}\right) \tilde{g}\left(J e_{b}{ }^{*}, e_{a}{ }^{*}\right), \\
& 4 R^{\perp}{ }_{i b a^{*} r}-4 \tilde{g}\left(\left[A_{a^{*}}, A_{r}\right] e_{i}, e_{b}\right)=-\left\|F e_{b}\right\| P\left(J e_{i}, e_{r}\right) \delta_{b a}, \\
& 2 R^{\perp}{ }_{i a s r}-2 \tilde{g}\left(\left[A_{s}, A_{r}\right] e_{i}, e_{a}\right)=P\left(J e_{i}, e_{a}\right) \tilde{g}\left(J e_{s}, e_{r}\right), \\
& 4 R^{\perp} d_{c b^{*} a^{*}}-4 \tilde{g}\left(\left[A_{b^{*}}, A_{a^{*}}\right] e_{d}, e_{c}\right)= \\
& =c\left\{\left\|F e_{c}\right\|\left\|F e_{d}\right\|\left(\delta_{d a} \delta_{c b}-\delta_{d b} \delta_{c a}\right)-2 \tilde{g}\left(T e_{d}, e_{c}\right) \tilde{g}\left(J e_{b}{ }^{*}, e_{a}{ }^{*}\right)\right\}- \\
& \text { - }\left\|F e_{c}\right\|\left\{P\left(J e_{d}, e_{a}{ }^{*}\right) \delta_{c b}-P\left(J e_{d}, e_{b}{ }^{*}\right) \delta_{c a}\right\}- \\
& -\left\|F e_{d}\right\|\left\{P\left(e_{c}{ }^{*}, e_{b}{ }^{*}\right) \delta_{d a}-P\left(J e_{c}, e_{a}{ }^{*}\right) \delta_{c b}\right\}+ \\
& +2\left\{\left\|F e_{c}\right\| P\left(J e_{d}, e_{c}\right) \tilde{g}\left(J e_{b}{ }^{*}, e_{a}{ }^{*}\right)+P\left(J e_{b}{ }^{*}, e_{a}{ }^{*}\right) \tilde{g}\left(T e_{d}, e_{c}\right)\right\} \text {, } \\
& 4 R^{\perp}{ }_{c b a^{*} r}-4 \tilde{g}\left(\left[A_{a^{*}}, A_{r}\right] e_{c}, e_{b}\right)=2 P\left(J e_{a}{ }^{*}, e_{r}\right) \tilde{g}\left(T e_{c}, e_{b}\right)+ \\
& +\left\|F e_{c}\right\|\left\|F e_{b}\right\|\left\{P\left(J e_{b}^{*}, e_{r}\right) \delta_{c a}-P\left(J e_{c}{ }^{*}, e_{r}\right) \delta_{b a}\right\}, \\
& 2 R^{\perp}{ }_{b a s r}-2 \tilde{g}\left(\left[A_{s}, A_{r}\right] e_{b}, e_{a}\right)=-c \tilde{g}\left(T e_{b}, e_{a}\right) \tilde{g}\left(J e_{r}, e_{s}\right)+ \\
& +P\left(J e_{b}, e_{a}\right) \tilde{g}\left(J e_{s}, e_{r}\right)+P\left(J e_{s}, e_{r}\right) \tilde{g}\left(T e_{b}, e_{a}\right),
\end{aligned}
$$

where $F e_{a}=\left\|F e_{a}\right\| a^{*}, j, i \in\{1,2, \ldots, 2 p\}, d, c, b, a \in\{2 p+1,2 p+2,2 p+q\}$, and $s, r \in\{n+q, n+q+1, \ldots, m\}$. By virtue of (2.5), we can formally put

$$
\begin{aligned}
J e_{j} & =\sum_{i=1}^{p}\left(T_{j}{ }^{i} e_{i}+T_{j}{ }^{p+i} e_{p+i}\right)+T_{j}{ }^{a} e_{a}+F_{j}{ }^{a} e_{a}^{*}+F_{j}{ }^{s} e_{s}, \\
J e_{p+j} & =\sum_{i=1}^{p}\left(T_{p+j}^{i} e_{i}+T_{p+j}^{p+i} e_{p+i}\right)+T_{p+j}^{a} e_{a}+F_{p+j}^{a} e_{a}{ }^{*}+F_{p+j}{ }^{s} e_{s}, \\
J e_{a} & =\sum_{i=1}^{p}\left(T_{a}{ }^{i} e_{i}+T_{a}{ }^{p+i} e_{p+i}\right)+T_{a}{ }^{c} e_{c}+F_{a}{ }^{c} e_{c}{ }^{*}+F_{a}{ }^{s} e_{s}, \\
J e_{a}{ }^{*} & =\sum_{i=1}^{p}\left(t_{a}{ }^{i} e_{i}+t_{a}{ }^{p+i} e_{p+i}\right)+t_{a}{ }^{c} e_{c}+f_{a}{ }^{c} e_{c}{ }^{*}+f_{a}{ }^{s} e_{s},
\end{aligned}
$$




$$
J e_{s}=\sum_{i=1}^{p}\left(t_{s}^{i} e_{i}+t_{s}^{p+i} e_{p+i}\right)+t_{s}{ }^{a} e_{a}+f_{s}{ }^{a} e_{a}{ }^{*}+f_{s}{ }^{r} e_{r}
$$

for $a, c \in\{2 p+1,2 p+2, \ldots, 2 p+q\}, s, r \in\{n+q+1, n+q+2, \ldots, m\}$, and $j \in\{1,2, \ldots, p\}$. Since, our frame is a generalized adapted one, we know in the above equation

$$
\begin{aligned}
& \left(\begin{array}{ccc}
T_{j}{ }^{i} & T_{p+j}^{i} & T_{a}{ }^{i} \\
T_{j}{ }^{p+i} & T_{p+j^{p+i}} & T_{a}{ }^{p+i} \\
T_{j}{ }^{a} & T_{p+j}^{a} & T_{b}{ }^{a}
\end{array}\right)=\left(\begin{array}{ccc}
0 & -\delta_{j}{ }^{i} & 0 \\
\delta_{j}{ }^{i} & 0 & 0 \\
0 & 0 & T_{b}{ }^{a}
\end{array}\right), \\
& \left(\begin{array}{cc}
F_{j}^{a} & F_{j}^{s} \\
F_{p+j}^{a} & F_{p+j}{ }^{s} \\
F_{b}{ }^{a} & F_{b}^{s}
\end{array}\right)=\left(\begin{array}{cc}
0 & 0 \\
0 & 0 \\
F_{b}{ }^{a} & 0
\end{array}\right) \\
& \left(\begin{array}{ccc}
t_{a}{ }^{i} & t_{a}{ }^{p+i} & t_{a}{ }^{c} \\
t_{s}{ }^{i} & t_{s}{ }^{p+i} & t_{s}{ }^{a}
\end{array}\right)=\left(\begin{array}{lll}
0 & 0 & 0 \\
0 & 0 & 0
\end{array}\right)
\end{aligned}
$$

and

$$
\left(\begin{array}{cc}
f_{a}{ }^{c} & f_{a}{ }^{s} \\
f_{s}{ }^{a} & f_{s}{ }^{r}
\end{array}\right)=\left(\begin{array}{cc}
0 & 0 \\
0 & f_{s}^{r}
\end{array}\right) .
$$

From (5.5), we can easily get that $f_{b a}=0$ identically.

Theorem 5.1. With respect to the generalized adapted frame, the tensor field $T, F, t$ and $f$ satisfy (5.2), (5.3), (5.4) and (5.5), respectively.

Thus due to (5.3), (5.4) and (5.5) the system of equations (5.1) can be written as follows:

$$
\begin{aligned}
& 2 R_{j i b^{*} a^{*}}-2 \tilde{g}\left(\left[A_{b^{*}}, A_{a^{*}}\right] e_{j}, e_{i}\right)=0, \\
& R^{\perp}{ }_{j i a^{*} r}-\tilde{g}\left(\left[A_{a^{*}}, A_{r}\right] e_{j}, e_{i}\right)=0, \\
& R^{\perp}{ }_{j i s r}-\tilde{g}\left(\left[A_{s}, A_{r}\right] e_{j}, e_{i}\right)=0, \\
& 4 R^{\perp}{ }_{i c b^{*} a^{*}}-4 \tilde{g}\left(\left[A_{b^{*}}, A_{a^{*}}\right] e_{i}, e_{c}\right)=\left\|F e_{c}\right\|\left(P_{i^{*} a^{*}} \delta_{c b}-P_{i^{*} b^{*}} \delta_{c a}\right), \\
& 4 R_{i b a^{*} r}^{\perp}-4 \tilde{g}\left(\left[A_{a^{*}}, A_{r}\right] e_{i}, e_{b}\right)=\left\|F e_{b}\right\| P_{i^{*} r} \delta_{b a}, \\
& 2 R_{i a s r}^{\perp}-2 \tilde{g}\left(\left[A_{s}, A_{r}\right] e_{i}, e_{a}\right)=-P_{i^{*} a} \tilde{g}\left(J e_{s}, e_{r}\right), \\
& 4 R^{\perp}{ }_{d c b^{*} a^{*}}-4 \tilde{g}\left(\left[A_{b^{*}}, A_{a^{*}}\right] e_{d}, e_{c}\right)=c\left\|F e_{c}\right\|\left\|F e_{d}\right\|\left(\delta_{d a} \delta_{c b}-\delta_{d b} \delta_{c a}\right)+ \\
& \quad+\left\|F e_{c}\right\|\left\{\left(T_{d}^{e} P_{e a^{*}}+F_{d}^{e} P_{e^{*} a^{*}}\right) \delta_{c b}-\left(T_{d}^{e} P_{e b^{*}}+F_{d}^{e} P_{e^{*} b^{*}}\right) \delta_{c a}\right\}- \\
& \quad-\left\|F e_{d}\right\|\left\{P_{c^{*} b^{*}} \delta_{d a}-\left(T_{c}^{e} P_{e a^{*}}+F_{c}{ }^{e} P_{e^{*} a^{*}}\right) \delta_{c b}\right\}, \\
& 4 R^{\perp}{ }_{c b a^{*} r}-4 \tilde{g}\left(\left[A_{a^{*}}, A_{r}\right] e_{c}, e_{b}\right)=0, \\
& 2 R_{b a s r}^{\perp}-2 \tilde{g}\left(\left[A_{s}, A_{r}\right] e_{b}, e_{a}\right)=-c T_{b a} \tilde{g}\left(J e_{r}, e_{s}\right)+F_{b}^{e} P_{e^{*} a} \tilde{g}\left(J e_{s}, e_{r}\right)+ \\
& \quad+P\left(J e_{s}, e_{r}\right) T_{b a},
\end{aligned}
$$


for $j, i \in\{1,2, \ldots, p\}, d, c, b, a \in\{2 p+1,2 p+2, \ldots, 2 p+q=n\}$ and $s, r \in\{n+q, n+q+1, . ., m\}$. Thus we have

Theorem 5.2. If the first type warped product semi-slant submanifold in an l.c.K.-space form $\tilde{M}(c)$ is normally flat, that is, $R^{\perp}=0$, identically, then the shape operators $A_{\lambda}$ satisfy

$$
\begin{aligned}
& \tilde{g}\left(\left[A_{b^{*}}, A_{a^{*}}\right] e_{j}, e_{i}\right)=0, \\
& \tilde{g}\left(\left[A_{a^{*}}, A_{r}\right] e_{j}, e_{i}\right)=0, \\
& \tilde{g}\left(\left[A_{s}, A_{r}\right] e_{j}, e_{i}\right)=0, \\
& 4 \tilde{g}\left(\left[A_{b^{*}}, A_{a^{*}}\right] e_{i}, e_{c}\right)=-\left\|F e_{c}\right\|\left(P_{i^{*} a^{*}} \delta_{c b}-P_{i^{*} b^{*}} \delta_{c a}\right), \\
& 4 \tilde{g}\left(\left[A_{a^{*}}, A_{r}\right] e_{i}, e_{b}\right)=-\left\|F e_{b}\right\| P_{i^{*} r} \delta_{b a}, \\
& 2 \tilde{g}\left(\left[A_{s}, A_{r}\right] e_{i}, e_{a}\right)=P_{i^{*} a} \tilde{g}\left(J e_{s}, e_{r}\right), \\
& 4 \tilde{g}\left(\left[A_{b^{*}}, A_{a^{*}}\right] e_{d}, e_{c}\right)=-c\left\|F e_{c}\right\|\left\|F e_{d}\right\|\left(\delta_{d a} \delta_{c b}-\delta_{d b} \delta_{c a}\right)+ \\
& +\left\|F e_{c}\right\|\left\{\left(T_{d}^{e} P_{e a^{*}}+F_{d}^{e} P_{e^{*} a^{*}}\right) \delta_{c b}-\left(T_{d}^{e} P_{e b^{*}}+F_{d}^{e} P_{e^{*} b^{*}}\right) \delta_{c a}\right\}- \\
& -\left\|F e_{d}\right\|\left\{P_{c^{*} b^{*}} \delta_{d a}-\left(T_{c}^{e} P_{e a^{*}}+F_{c}^{e} P_{e^{*} a^{*}}\right) \delta_{c b}\right\}, \\
& \tilde{g}\left(\left[A_{a^{*}}, A_{r}\right] e_{c}, e_{b}\right)=0, \\
& 2 \tilde{g}\left(\left[A_{s}, A_{r}\right] e_{b}, e_{a}\right)=c T_{b a} \tilde{g}\left(J e_{r}, e_{s}\right)-F_{b}{ }^{e} P_{e^{*} a} \tilde{g}\left(J e_{s}, e_{r}\right)-P\left(J e_{s}, e_{r}\right) T_{b a},
\end{aligned}
$$

\section{ACKNOWLEDGEMENTS}

The author deeply thanks to Professor Yevhen Cherevko at Odessa National Economic University for his very kind support.

\section{REFERENCES}

[1] Aurel Bejancu. CR submanifolds of a Kaehler manifold. II. Trans. Amer. Math. Soc., 250:333-345, 1979.

[2] Aurel Bejancu. Geometry of CR-submanifolds, volume 23 of Mathematics and its Applications (East European Series). D. Reidel Publishing Co., Dordrecht, 1986.

[3] Vittoria Bonanzinga, Koji Matsumoto. Warped product CR-submanifolds in locally conformal Kaehler manifolds. Period. Math. Hungar., 48(1-2):207-221, 2004.

[4] Bang-yen Chen. Geometry of submanifolds. Marcel Dekker, Inc., New York, 1973. Pure and Applied Mathematics, No. 22.

[5] Bang-yen Chen. CR-submanifolds of a Kaehler manifold. I. J. Differential Geom., 16(2):305-322, 1981.

[6] Bang-yen Chen. CR-submanifolds of a Kaehler manifold. II. J. Differential Geom., 16(3):493-509 (1982), 1981.

[7] Bang-Yen Chen. Geometry of slant submanifolds. Katholieke Universiteit Leuven, Louvain, 1990. 
[8] Toyoko Kashiwada. Some properties of locally conformal Kähler manifolds. Hokkaido Math. J., 8(2):191-198, 1979.

[9] Viqar Azam Khan, Meraj Ali Khan. Semi-slant submanifolds of a nearly Kaehler manifold. Turkish J. Math., 31(4):341-353, 2007.

[10] Koji Matsumoto. On CR-submanifolds of locally conformal Kähler manifolds. J. Korean Math. Soc., 21(1):49-61, 1984.

[11] Koji Matsumoto. Warped product semi-slant submanifolds in locally conformal Kaehler manifolds. Proc. Int. Geom. Cent., 10(2):8-23, 2017.

[12] Barrett O'Neill. Semi-Riemannian geometry, volume 103 of Pure and Applied Mathematics. Academic Press, Inc. [Harcourt Brace Jovanovich, Publishers], New York, 1983. With applications to relativity.

[13] Neculai Papaghiuc. Semi-slant submanifolds of a Kaehlerian manifold. An. Ştiinţ. Univ. Al. I. Cuza Iaşi Secţ. I a Mat., 40(1):55-61, 1994.

[14] Izu Vaisman. On locally conformal almost Kähler manifolds. Israel J. Math., 24(34):338-351, 1976.

Received: August 7, 2018, accepted: October 19, 2018.

Koji Matsumoto

Yamagata University, 2-3-65 Nishi-Odori, Yonezawa, Yamagata, 992-0059, JaPAN

Email: tokiko_matsumoto@yahoo.com 